\title{
The co-application of hypoxic preconditioning and postconditioning abolishes their own protective effect on systolic function in human myocardium
}

\author{
Tomasz Roleder ${ }^{1}$, Krzysztof S. Gołba ${ }^{1}$, Marcin Kunecki ${ }^{1}$, Marcin Malinowski ${ }^{2}$, \\ Jolanta Biernat ${ }^{1}$, Grzegorz Smolka ${ }^{3}$, Marek A. Deja ${ }^{2}$ \\ ${ }^{1}$ Department of Cardiology, Medical University of Silesia, Katowice, Poland \\ ${ }^{2} 2^{\text {nd }}$ Department of Cardiac Surgery, Medical University of Silesia, Katowice, Poland \\ ${ }^{3} 3^{\text {rd }}$ Department of Cardiology, Medical University of Silesia, Katowice, Poland
}

\begin{abstract}
Background: Ischemic preconditioning (IPC) and postconditioning (POC) are well documented to trigger cardioprotection against ischemia/reperfusion $(I / R)$ injury, but the effect of their both co-application remains unclear in human heart. The present study sought to assess the co-application of IPC and POC on fragments of human myocardium in vitro.

Methods: Muscular trabeculae of the human right atrial were electrically driven in the organ bath and subjected to simulated I/R injury - hypoxia/re-oxygenation injury in vitro. To achieve IPC of trabeculae the single brief hypoxia period preceded the applied lethal hypoxia, and to achieve POC triple brief hypoxia periods followed the lethal hypoxia. Additional muscular trabeculae were exposed only to the hypoxic stimulation (Control) or were subjected to the non-hypoxic stimulation (Sham). $10 \mu M$ norepinephrine (NE) application ended every experiment to assess viability of trabeculae. The contraction force of the myocardium assessed as a maximal amplitude of systolic peak $\left(\% A_{\max }\right)$ was obtained during the whole experiment's period.
\end{abstract}

Results: Co-application of IPC and POC resulted in decrease in $\% A_{\max }$ during the re-oxygentaion period and after NE application, as compared to Control (30.35 \pm 2.25 vs. $41.89 \pm 2.25$, $56.26 \pm 7.73$ vs. $65.98 \pm 5.39$, respectively). This was in contrary to the effects observed when IPC and POC were applied separately.

Conclusions: The co-application of IPC and POC abolishes the cardioprotection of either intervention alone against simulated $I / R$ injury in fragments of the human right heart atria. (Cardiol J 2013; 20, 5: 472-477)

Key words: ischemia, reperfusion, preconditioning, postconditioning

\section{Introduction}

The restoration of coronary flow is necessary to reduce ischemic myocardial damage due to acute vessel occlusion. However, during the reperfusion period, an additional damage of cardiac tissue occurs resulting in an ischemia/reperfusion (I/R) injury [1]. I/R injury causes a necrosis and apoptosis of cardiomyocytes and in clinical settings it may manifests as a myocardial stunning observed after the global ischemia during the cardiac surgery [2] or as an impaired coronary flow (no-flow pheno-

Address for correspondence: Tomasz Roleder, MD, PhD, The Medical University of Silesia in Katowice, ul. Poniatowskiego 15, 40-055 Katowice, e-mail: tomaszroleder@gmail.com 
menon) [3] due to microvessels dysfunction observed after the percutaneous coronary angioplasty.

Sequences of brief ischemia periods applied before (preconditioning — IPC) or after (postconditioning - POC) the prolonged coronary occlusion was documented to protect the human heart against $\mathrm{I} / \mathrm{R}$ injury in in vitro $[4,5]$ and in in vivo studies [6-10]. Since IPC and POC act at the opposite stages of ischemia, thus the question arise if their protective capacity is similar and if their co-application can ensure better overall cardioprotection against I/R injury in human heart.

We aimed to assess the influence of IPC and POC co-application on the simulated I/R injury outcomes in the human myocardium. Hence in the following study, we have tested several protocols of IPC to identify the one producing the maximal protection. Such selected IPC protocol was than combined with the POC protocol that proved to be cardioprotective in previous studies $[9,10]$.

\section{Methods}

The experiments were performed on muscular trabeculae obtained from the right heart atrial appendages of 44 consecutive patients (32 males/ $/ 12$ females) subjected to the coronary artery bypass surgery. Patients with diagnosed diabetes, a significant valvular heart disease and subjected to oral statin therapy were excluded from the study.

The fragments of the human right heart atria were transported from the cardiac surgery room to the laboratory in an ice-cold Krebs-Henseleit solution ([M]: $\mathrm{NaCl} 118.0, \mathrm{KCl} 4.70, \mathrm{CaCl}_{2} 1.52$, $\mathrm{MgSO}_{4}$ 1.64, $\mathrm{NaHCO}_{3} 24.88, \mathrm{KH}_{2} \mathrm{PO}_{4}$ 1.18, glucose 11.0 , and sodium pyruvate $2.0 ; \mathrm{pH} 7.4$ ). Two muscular trabeculae, each less than $1 \mathrm{~mm}$ in diameter, were dissected from the 1 fragment of the right heart atria and incubated in 2 separate organ baths (Schuler Organbath, Hugo Sachs Elektronik, March-Hugstetten, Germany [HSE]) both filled with Krebs-Henseleit solution warmed up to $37^{\circ} \mathrm{C}$. Two trabeculae from 1 patient were always studied simultaneously using POC or IPC protocol and only hypoxia protocol (Control). Additional trabeculae were exposed to 120-min non-hypoxic stimulation in the sham protocol (Sham). All trabeculae, except those in Sham protocol, were exposed to hypoxia protocol including: $60 \mathrm{~min}$ of hypoxia (the trabecula was incubated in the Krebs-Henseleit buffer deprivated of glucose and pyruvate and saturated with 95\% argon and 5\% carbon dioxide) with subsequent 60 min of re-oxygenation (the trabecula was incubated in the stanadard Krebs-Henseleit buffer saturated with the $95 \%$ oxygen and $5 \%$ carbon dioxide). The buffer was replaced every $15 \mathrm{~min}$, except the time of hypoxia. Every trabecula was stretched to $90 \%$ of its optimal tension strength, according to the Frank-Starling relationship and all trabeculae were driven throughout experiments with $1 \mathrm{~Hz} 50 \mathrm{~ms}$ square stimuli using platinum field electrodes and a stimulator (Type 215, HSE) as described previously [11]. The systolic function of the every trabecula was recorded with the use an F30 isometric force transducer (Type 372, HSE). The signal was enhanced with a bridge amplifier (Type 336, HSE) and recorded by a PowerLab/4SP system and analyzed off-line using Chart software (AD Instruments, Chalgrove, Oxfordshire, UK).

POC protocol consisted of 3 times repeated sequence of 1-min re-oxygenation with subsequent 1-min hypoxia applied at the beginning of $60 \mathrm{~min}$ re-oxygenation during hypoxia protocol. The number of the ischemic cycles was based on previously published data $[9,10]$. Three experiments of IPC protocol were performed to identify the 1 producing maximal cadioprotection. The $1^{\text {st }}$ IPC protocol consisted of 5-min hypoxia with subsequent 5 -min of re-oxygenation applied before hypoxia protocol (IPC-1), the $2^{\text {nd }}$ consisted of 2 periods of 5 -min hypoxia with subsequent 5 -min of re-oxygenation applied before hypoxia protocol (IPC-2) and $3^{\text {rd }}$ consisted of 3 periods of $5-\mathrm{min}$ hypoxia with subsequent 5 -min of re-oxygenation applied before hypoxia protocol (IPC-3). The last experimental protocol consisted of combined IPC and POC protocols (IPC-2 + POC) applied in one trabecula simultaneously.

Each experimental protocol was completed with $10 \mu \mathrm{M}$ of the norepinephrine (NE) application. The level of the time depending on decline of the myocardial systolic function in our bio-assay setting was the net effect of necrosis and/or apoptosis in incubated myocardial strip which leads to the loss of its viability. The percentage recovery of the myocardial function obtained after NE application was the measure of (1) tissue viability at the end of protocol and (2) the achieved by protocol cardioprotection [12].

The systolic function was assessed by the measurement of the maximal amplitude of the systolic peak $\left(\mathrm{A}_{\max }[\mathrm{mV}]\right) . \mathrm{A}_{\max }$ was obtained in $10^{\text {th }}, 15^{\text {th }}, 30^{\text {th }}$, $45^{\text {th }}$ and $60^{\text {th }}$ min of re-oxygenation and after the NE application. The $\mathrm{A}_{\max }$ values were presented as the percent of $\mathrm{A}_{\max }$ obtained before any experimental protocol application $\left(\% \mathrm{~A}_{\max }\right)$.

The Local Bioethics Committee approval for the use of human tissue was obtained and individual 
Table 1. The patients' demographic information and the preoperative pharmacotherapy.

\begin{tabular}{lccccccc}
\hline & $\begin{array}{c}\text { Sham } \\
(\mathbf{n}=10)\end{array}$ & $\begin{array}{c}\text { IPC-1 } \\
(\mathbf{n}=12)\end{array}$ & $\begin{array}{c}\text { IPC-2 } \\
(\mathbf{n}=\mathbf{1 2})\end{array}$ & $\begin{array}{c}\text { IPC-3 } \\
(\mathbf{n}=12)\end{array}$ & $\begin{array}{c}\text { POC } \\
(\mathbf{n}=12)\end{array}$ & $\begin{array}{c}\text { IPC+POC } \\
(\mathbf{n}=8)\end{array}$ & P \\
\hline Age [years] & $64.8 \pm 3.7$ & $64.7 \pm 3.2$ & $58.5 \pm 2.7$ & $56.3 \pm 2.62$ & $63.5 \pm 4.3$ & $59.3 \pm 5.2$ & 0.262 \\
Male & $7(70 \%)$ & $8(68 \%)$ & $8(68 \%)$ & $9(75 \%)$ & $10(83 \%)$ & $7(88 \%)$ & 0.839 \\
Beta-blockers & $8(80 \%)$ & $10(83 \%)$ & $8(68 \%)$ & $9(75 \%)$ & $10(83 \%)$ & $8(100 \%)$ & 0.589 \\
ACEl/ARBII & $9(90 \%)$ & $9(75 \%)$ & $10(83 \%)$ & $7(58 \%)$ & $10(83 \%)$ & $5(63 \%)$ & 0.641 \\
CCB & $1(10 \%)$ & $2(17 \%)$ & $1(8 \%)$ & $3(25 \%)$ & $4(50 \%)$ & $4(33 \%)$ & 0.237 \\
\hline
\end{tabular}

IPC - preconditioning protocol with single (IPC-1), twice repeated (IPC-2) or three times repeated brief periods of ischemia (IPC-3); $\mathrm{POC}$ - postconditioning protocol; beta-blockers - beta-adrenergic receptor blockers; ACEI — angiotensin converting enzyme inhibitros; ARBII - angiotensin II receptor blockers; CCB - calcium channel blockers

patient consent was waived. All experiments were performed according to the principles stated in the Declaration of Helsinki.

\section{Statistical analysis}

All continuous data were normally distributed and were presented as a mean \pm standard error of the mean (SEM). Chi-square analysis were used to compare categorical data of patients' characteristic. Two-way ANOVA was used to compare the results of $\% \mathrm{~A}_{\max }$ values from $10^{\text {th }}$ to $60^{\text {th }}$ min of re-oxygenation. Paired Student's t-test was performed to compare patients' age and $\% \mathrm{~A}_{\max }$ values obtained in the $60^{\text {th }}$ min re-oxygenation or after NE application.

A substraction sum of \%Amax values between IPC-1, IPC-2, POC and corresponding control were evaluated to assess differences in the myocardial response to used experimental protocols (dif-IPC-1, dif-IPC-2 and dif-POC respectively). dif-IPC-1, dif-IPC-2 and dif-POC results were compared by 2 -way ANOVA for values obtained together between $10^{\text {th }}$ to $60^{\text {th }}$ min of re-oxygenation and by unpaired Student t-test for values obtained in the $60^{\text {th }}$ min of re-oxygenation or after NE application (SigmaPlot 10.0.1.2., Systat Software Inc.).

\section{Results}

\section{Patients' characteristics}

There were no significant differences in age, sex and pharmacotherapy between patients subjected to Sham, IPC-1, IPC-2, IPC-3 POC and IPC + POC protocols (Table 1).

\section{Preconditioning}

Maximal amplitude of systolic peak values were significantly higher for IPC-1 and IPC-2 protocols vs. Control from $10^{\text {th }}$ to $60^{\text {th }}$ min of re-oxygenation and after NE application. However, in the $60^{\text {th }}$ min of re-oxygenation, $\% \mathrm{~A}_{\max }$ values were higher only for IPC-1 vs. Control. No difference was found between IPC- $3 \% \mathrm{~A}_{\max }$ values vs. Control (Fig. 1). Moreover, dif-IPC-1\% $\mathrm{A}_{\max }$ values were significantly higher as compared to dif-IPC-2 analyzed together in $10^{\text {th }}$ to $60^{\text {th }}$ min of re-oxygenation $(10.7 \pm 1.82$ vs. $7.5 \pm 1.37 ; \mathrm{p}<0.05)$.

\section{Postconditioning}

Maximal amplitude of systolic peak values were significantly higher for POC vs. Control from $10^{\text {th }}$ to $60^{\text {th }}$ min of re-oxygenation, in the $60^{\text {th }} \mathrm{min}$ of re-oxygenation and after NE application (Fig. 1). There were no significant differences between dif-IPC 1 and dif-POC $(10.7 \pm 1.82$ vs. $11.58 \pm 1.69$; $\mathrm{p}<0.005)$.

\section{Co-application of preconditioning and postconditioning}

Maximal amplitude of systolic peak values were significantly lower for IPC-1 + POC vs. Control from $10^{\text {th }}$ to $60^{\text {th }}$ min of re-oxygenation and in the $60^{\text {th }}$ min of re-oxygenation and after NE application.

\section{Sham}

Maximal amplitude of systolic peak values were significantly higher for Sham vs. Control from $10^{\text {th }}$ to $60^{\text {th }}$ min of re-oxygenation and after $\mathrm{NE}$ application. However the $\% \mathrm{~A}_{\max }$ were gradually falling in Sham and were no different from control in $60^{\text {th }}$ min of re-oxygenation (Fig. 1).

\section{Discussion}

Previous studies utilized animal models to describe the influence of IPC and POC co-application on I/R injury. Our study is the first to assess the influence of IPC and POC interaction on hypoxic human myocardium in vitro. The main finding of our study is that co-application of IPC and POC abolished the cardioprotective effect of either intervention alone in fragments of human right heart atria. 


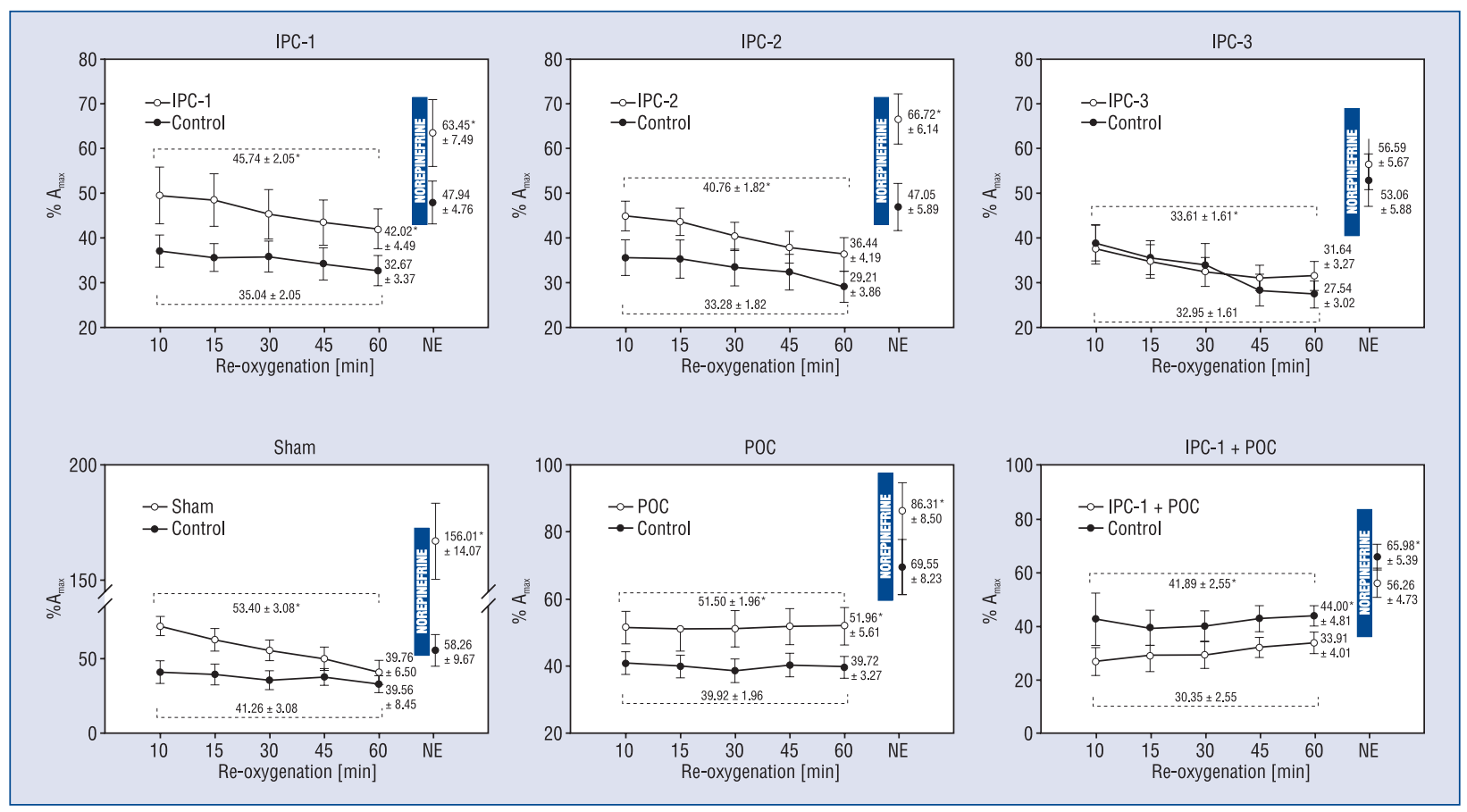

Figure 1. The myocardial strips function during the re-oxygenation period. Figures present maximal amplitude of systolic peak $\left(\% \mathrm{~A}_{\max }\right)$ results analysis by two-way ANOVA during within $10^{\text {th }}$ to $60^{\text {th }}$ min of re-oxygenation and by paired t-Student's test in the $60^{\text {th }}$ min of re-oxygenation and after norepinephrine (NE) application $\left({ }^{*} p<0.05\right.$ vs. Control) for IPC-1 (preconditioning using single hypoxia/re-oxygenation period), IPC-2 (preconditioning using twice repeated hypoxia/re-oxygenation period), IPC-3 (preconditioning using three times repeated hypoxia/re-oxygenation period), POC (postconditioning using three times repeated hypoxia/re-oxygenation period) and the combined use of IPC-1 and POC. Sham refers to non-hypoxic myocardial strips incubation. As Control serves results of hypoxia/re-oxygenation injury of myocardial strips.

Our results are in the contrast to previous reports. Animal experiments presented that additional POC application did not diminish the cardioprotection achieved by IPC alone. IPC and POC co-applied in myocardium subjected to 60 min of lethal ischemia was protective against $I / R$ injury and the reduction of the infract size was equal to the one achieved by either intervention alone [10, 13-15]. Interestingly, the reduction of lethal ischemia time to 45 or 30 min resulted in a greater infract size reduction for IPC and POC co-application, as compared to the either intervention alone $[13,15]$. Such previously reported outcomes of IPC and POC interaction were not depended on the used study protocol. The single brief IPC ischemia period lasted from 3 to 15 min and was repeated up to 3 times, and $\mathrm{POC}$ ischemia period lasted 10 to $30 \mathrm{~s}$ and was repeated from 3 to 6 times after lethal ischemia [10, 13-15].

We observed that performed IPC and POC protocols in our study successfully protected the human myocardium against simulated I/R injury, and the level their protection was similar. This is in a line with previous findings, where those 2 endogenous protective mechanisms were equal to each other $[7,10,16,17]$. Moreover, we have confirmed that single brief period of ischemia before lethal ischemia injury is the most effective IPC algorithm to protect the human heart in vitro [4].

The results of our study shed a light on the IPC and POC interaction in human myocardium and may help to explain previously observed discrepancies between promising results of ischemic POC application in experimental models $[5,17]$ and its less optimistic clinical outcomes. Clinical trials reported conflicting results of $\mathrm{POC}$ application during the percutaneous coronary intervention of the infarct related artery. POC triggered cardioprotection [8] or did not protect myocardium against I/R injury at all [18]. Such observed discrepancies may be related to diverse scenarios of coronary artery occlusion. Coronary artery may occlude abruptly or may occlude progressively with intermittent coronary flow limitations mimicking IPC $[19,20]$. These 2 different scenarios of the myocardial ischemia onset may cause dissimilar 
outcomes of POC application during the percutaneous coronary intervention of the infarct related artery. As it was shown in our study, POC application in the myocardium subjected to IPC may abolish overall cardioprotection. Thus, POC application in patients with the intermittent ischemia symptoms suggesting IPC may not be beneficial and POC application may only be effective in patients without pre-infarct angina symptoms. Moreover, further investigations are needed to determine whether patients' age influences the IPC and POC interaction outcomes. As it was presented in animal studies, IPC and POC co-application was not detrimental to either intervention alone in relatively young study population $[10,13-15]$ what is in a contrast to IPC and POC co-application outcomes in our aging study group [21].

We may only speculate on the mechanism in which the co-application of IPC and POC get toxic for the hypoxic human heart. Previous data presented that both IPC and POC cardioprotection are related to the activity of the nitric oxide synthase and nitric oxide (NO) concentration [22, 23]. Although NO at low concentration triggered the cardioprotection of human heart [24], its protective capacity was lost at high concentration due to the increase of free radicals concentration; especially the peroxynitrite [25]. It is plausible that, the co-application of both IPC and POC increases NO concentration to a level that it triggers the excessive peroxinitrite production, which is toxic for human heart subjected to I/R injury.

\section{Limitations of the study}

Firstly, we utilized fragments of human right heart atria; not fragments of the left heart ventricle. Previous histological studies showed that the atrial wall is covered by thicker layer of the endothelium, as compared to the heart ventricle's wall [26]. Thicker endothelium is a source of a higher rate of NO synthesis in atria, as compared to ventricles [27]. An increased NO synthesis was shown to protect the myocardium against I/R injury [28], and alterations in endothelial NO concentration was documented to influence IPC and POC outcomes $[22,23]$. Such histological differences followed by dissimilar NO synthesis rate may cause a different atrial myocardium response to IPC and POC stimuli, as compared to ventricular myocardium.

Secondly, all of the patients received preoperative (pre-experimental) pharmacotherapy, what is in contrast to the above-mentioned in vitro animal experiments. However, we excluded patients subjected to statin therapy to avoid its detrimental effect on IPC and POC protection [29].
Thirdly, we observed a relatively rapid decline in the systolic function of myocardium not subjected to $\mathrm{I} / \mathrm{R}$ injury what might indicate an intra-experimental myocardial stunning of non-hypoxic trabeculae. This calls doubts about the low stability of presented bio-assay. But the intention was to carry out experiment so long to achieve a decline in function in all groups hypoxic and non-hypoxic (Sham). This approach gave a chance for a better comparison of viability by the use of NE stimuli. We found in Sham 3 fold higher viability than in Control. So we presented high stability of our bioassay in terms of myocardial tissue viability.

Finally, we have only assessed the myocardial function and did not assess the rate of myocardial necrosis and/or apoptosis.

\section{Conclusions}

A combined application of IPC and POC protocols abolishes their protective capacity, as compared to the either intervention alone in fragments of human right atria. A thorough knowledge about IPC and POC interactions in the human heart is needed to design the most suitable method of their effective application in clinical settings.

\section{References}

1. Becker LC, Ambrosio G. Myocardial consequences of reperfusion. Prog Cardiovasc Dis, 1987; 30: 23-44.

2. Bolli R, Jeroudi MO, Patel BS et al. Marked reduction of free radical generation and contractile dysfunction by antioxidant therapy begun at the time of reperfusion. Evidence that myocardial "stunning" is a manifestation of reperfusion injury. Circ Res, 1989; 65: 607-622.

3. Kloner RA, Ganote CE, Jennings RB. The "no-reflow" phenomenon after temporary coronary occlusion in the dog. J Clin Invest, 1974; 54: 1496-1508.

4. Ghosh S, Standen NB, Galinanes M. Preconditioning the human myocardium by simulated ischemia: studies on the early and delayed protection. Cardiovasc Res, 2000; 45: 339-350.

5. Sivaraman V, Mudalagiri NR, Di Salvo C et al. Postconditioning protects human atrial muscle through the activation of the RISK pathway. Basic Res Cardiol, 2007; 102: 453-459.

6. Yellon DM, Alkhulaifi AM, Pugsley WB. Preconditioning the human myocardium. Lancet, 1993; 342: 276-277.

7. Alkhulaifi AM, Yellon DM, Pugsley WB. Preconditioning the human heart during aorto-coronary bypass surgery. Eur J Cardiothorac Surg, 1994; 8: 270-275.

8. Staat P, Rioufol G, Piot C et al. Postconditioning the human heart. Circulation, 2005; 112: 2143-2148.

9. Luo W, Li B, Chen R, Huang R, Lin G. Effect of ischemic postconditioning in adult valve replacement. Eur J Cardiothorac Surg, 2008; 33: 203-208.

10. Halkos ME, Kerendi F, Corvera JS et al. Myocardial protection with postconditioning is not enhanced by ischemic preconditioning. Ann Thorac Surg, 2004; 78: 961-969. 
11. Deja MA, Golba KS, Malinowski M et al. Diazoxide provides maximal KATP channels independent protection if present throughout hypoxia. Ann Thorac Surg, 2006; 81: 1408-1416.

12. Ellis SG, Wynne J, Braunwald E, Henschke CI, Sandor T, Kloner RA. Response of reperfusion-salvaged, stunned myocardium to inotropic stimulation. Am Heart J, 1984; 107: 13-19.

13. Manintveld OC, Hekkert ML, van der Ploeg NT, Verdouw PD, Duncker DJ. Interaction between pre- and postconditioning in the in vivo rat heart. Exp Biol Med (Maywood), 2009; 234: 1345-1354.

14. Tsang A, Hausenloy DJ, Mocanu MM, Yellon DM. Postconditioning: a form of "modified reperfusion" protects the myocardium by activating the phosphatidylinositol 3-kinase-Akt pathway. Circ Res, 2004; 95: 230-232.

15. Yang XM, Proctor JB, Cui L, Krieg T, Downey JM, Cohen MV. Multiple, brief coronary occlusions during early reperfusion protect rabbit hearts by targeting cell signaling pathways. J Am Coll Cardiol, 2004; 44: 1103-1110.

16. Gao X, Ren C, Zhao H. Protective effects of ischemic postconditioning compared with gradual reperfusion or preconditioning. J Neurosci Res, 2008; 86: 2505-2511.

17. Ma XJ, Zhang XH, Luo M, Li CM, Shao JH. Effects of preconditioning and postconditioning on emergency percutaneous coronary intervention in patients with acute myocardial infarction. Zhonghua Yi Xue Za Zhi, 2007; 87: 114-117.

18. Freixa $\mathrm{X}$, Bellera N, Ortiz-Perez JT et al. Ischaemic postconditioning revisited: lack of effects on infarct size following primary percutaneous coronary intervention. Eur Heart J, 2012; 33: 103-112.

19. Falk E. Unstable angina with fatal outcome: dynamic coronary thrombosis leading to infarction and/or sudden death. Autopsy evidence of recurrent mural thrombosis with peripheral embolization culminating in total vascular occlusion. Circulation, 1985; 71: 699-708.
20. Ottani F, Galvani M, Ferrini D et al. Prodromal angina limits infarct size. A role for ischemic preconditioning. Circulation, 1995; 91: 291-297.

21. Fenton RA, Dickson EW, Meyer TE, Dobson JG, Jr. Aging reduces the cardioprotective effect of ischemic preconditioning in the rat heart. J Mol Cell Cardiol, 2000; 32: 1371-1375.

22. Lochner A, Marais E, Genade S, Moolman JA. Nitric oxide: A trigger for classic preconditioning? Am J Physiol Heart Circ Physiol, 2000; 279: H2752-H2765.

23. Penna C, Cappello S, Mancardi D et al. Post-conditioning reduces infarct size in the isolated rat heart: Role of coronary flow and pressure and the nitric oxide/cGMP pathway. Basic Res Cardiol, 2006; 101: 168-179.

24. Trochu JN, Bouhour JB, Kaley G, Hintze TH. Role of endothelium-derived nitric oxide in the regulation of cardiac oxygen metabolism: implications in health and disease. Circ Res, 2000; 87: 1108-1117.

25. Radi R, Rodriguez M, Castro L, Telleri R. Inhibition of mitochondrial electron transport by peroxynitrite. Arch Biochem Biophys, 1994; 308: 89-95.

26. McMillan JB LM. The aging heart. I. Endocardium. J Gerontol, 1959; 14: 268-283.

27. Khadour FH, O'Brien DW, Fu Y, Armstrong PW, Schulz R. Endothelial nitric oxide synthase increases in left atria of dogs with pacing-induced heart failure. Am J Physiol, 1998; 275: H1971$-\mathrm{H} 1978$.

28. Bolli R. Cardioprotective function of inducible nitric oxide synthase and role of nitric oxide in myocardial ischemia and preconditioning: an overview of a decade of research. J Mol Cell Cardiol, 2001; 33: 1897-1918.

29. Kocsis GF, Pipis J, Fekete V et al. Lovastatin interferes with the infarct size-limiting effect of ischemic preconditioning and postconditioning in rat hearts. Am J Physiol Heart Circ Physiol, 2008; 294: H2406-H2409. 